\title{
Endoscopic endonasal repair with polyethylene implants in medial orbital wall fractures: A prospective study on 25 cases
}

\author{
Giacomo Colletti a, Alberto Maria Saibene ${ }^{\mathrm{b}}$, Lorenzo Giannini ${ }^{\mathrm{b}, ~ *}$, Margherita Dessy ${ }^{\mathrm{a}}$, \\ Alberto Deganello ${ }^{\mathrm{c}}$, Carlotta Pipolo ${ }^{\mathrm{b}}$, Fabiana Allevi a, Paolo Lozza ${ }^{\mathrm{b}}$, Giovanni Felisati ${ }^{\mathrm{b}}$, \\ Federico Biglioli ${ }^{\text {a }}$
}

${ }^{a}$ Unit of Maxillo-Facial Surgery, Department of Head \& Neck Surgery, ASST Santi Paolo e Carlo, Department of Health Sciences,

Università degli Studi di Milano, Milan, Italy

${ }^{\mathrm{b}}$ Unit of Otolaryngology, Department of Head \& Neck Surgery, ASST Santi Paolo e Carlo, Department of Health Sciences,

Università degli Studi di Milano, Milan, Italy

${ }^{\mathrm{c}}$ Department of Surgery and Translational Medicine, University of Florence, Italy

\section{A R T I C L E I N F O}

Article history:

Paper received 24 July 2017

Accepted 14 November 2017

Available online $\mathrm{xxx}$

\section{Keywords:}

Orbital fractures

Lamina papyracea

Medial orbital wall

Orbital endonasal repair

\begin{abstract}
A B S T R A C T
The aim of the study was to assess the mid-term effectiveness and safety of an original technique consisting of reconstructing fractures of the medial wall of the orbit with porous polyethylene implants with an exclusive transnasal approach.

Twenty-five patients were treated. Each patient underwent a pre-operative ophthalmologic evaluation and a CT scan. The surgery started with an anteroposterior ethmoidectomy of the fractured side; all the fractured bone fragments were removed and all usual landmarks of healthy bony margins were identified. A Medpor sheet was placed endoscopically to reconstruct the fractured wall. Each patient received an immediate postoperative $\mathrm{CT}$ scan, and was evaluated at day 1, 7, 30 and 6 months after surgery clinically and with an endoscopic examination.

In all patients, preoperative enophthalmos and/or diplopia were corrected. The CT scans showed excellent reconstruction of the fractured bony walls. The immediate postoperative period was characterized by a very high degree of subjective comfort. No perioperative complications were detected. At the 6 months follow up, all meshes appeared covered by epithelialized mucosa at the endoscopic inspection, and clinical results were stable. Scars or lid complications are always prevented.

The technique described has become the standard to treat medial wall fractures in our department. ๑) 2017 Published by Elsevier Ltd on behalf of European Association for Cranio-Maxillo-Facial Surgery.
\end{abstract}

\section{Introduction}

The medial wall of the orbit is predominantly made by the orbital plate of the ethmoid, also called lamina papyracea; this is a very thin bony plate $(0.2-0.4 \mathrm{~mm}$ thick) that keeps the orbit and the ethmoidal cells separated (Joseph and Glavas, 2011).

Due to its fineness, lamina papyracea is frequently damaged in orbital medial wall fractures, making these at least as frequent as fractures of the floor of the orbit (Choi et al., 2015). The mechanisms that determine medial orbital wall blow-out fractures (BOF) are explained by two theories: the hydraulic theory and the buckling

\footnotetext{
* Corresponding author. Unit of Otolaryngology, Department of Head \& Neck Surgery, ASST Santi Paolo e Carlo Via di Rudinì 8, 20142, Milano, Italy.

E-mail address: gianninilorenzo88@gmail.com (L. Giannini).
}

theory (Bullock et al., 1999) The first one asserts that the energy exerted to the globe consequently elevates intraorbital pressure, transmitting the force to the orbital wall and causing the fracture in the locus minoris resistentiae; the buckling theory sustains that a direct trauma on the medial orbital rim causes a fracture of the medial wall (Bullock et al., 1999).

Thus, blunt traumas to the eyeball or to the medial rim of the orbit may fracture the lamina, leading to a herniation of medial orbital content in the nasal cavity (blow-out fracture) with possible onset of enophthalmos and/or diplopia (Kim et al., 2016). In case of entrapment of the medial rectus muscle, Pseudo-Duane retraction syndrome may take place, consisting of retraction of the globe during abduction of the fractured eye (Duane et al., 1976).

Surgical treatment of the fracture is indicated when any of the aforementioned complications are observed, namely diplopia and severe enophthalmos. 
Many different techniques have been proposed to approach and manage medial wall fractures (Damgaard et al., 2016). Historically, the medial wall was reached through a Lynch incision (Lynch, 1921). This is nowadays abandoned because it leads to poor aesthetic results. Other skin accesses may be used, such as the subciliary incision (Kahn and Varvares, 2006). Yet these provide a limited view, with the additional price of a skin incision. Transconjunctival approaches are the most traditionally adopted ones: these include the transcaruncular, precaruncular and retrocaruncular (Kempton et al., 2016). These allow direct and fast access to the fracture and a very good exposure of the anterior portion of the medial wall. Transconjunctival approaches are however hampered by a very limited visibility of the posterior and superior areas of the medial wall and by potential injuries to the lacrimal sac and to the lesser oblique muscle. The eyeball itself is handled during these procedures, making inadvertent conjunctival or corneal damage all but impossible.

In the last 25 years, the widespread adoption of transnasal endoscopic surgery has led several authors to manage medial wall fractures through this route (Jin et al., 2007; Bartoli et al., 2015; Copelli et al., 2015).

Usually however, all authors rely on packing the medial wall with a silicone sheet (mainly Silastic) and additional tampons for 2-5 weeks in order to contain the herniated orbital content (Jin et al., 2007). The consequences of this are an obvious discomfort for the patient and a theoretical increased risk of infection. Moreover, all these techniques do not allow for direct checking of fracture reduction and orbital tissue containment.

Our centre has recently introduced a pure endoscopic technique employing a porous polyethylene lamina to reconstruct the fractured wall (Medpor, Stryker, Kalamazoo, MI, USA) (Colletti et al., 2016).

The current paper presents the data of a prospective 25-patient case series where this technique was adopted. Short and long term results were considered. All potential complications were ruled out.

\section{Material and methods}

Twenty-five consecutive patients were enrolled in the present study

Inclusion criteria were:

1. A fracture of the sole medial wall of the orbit (22 patients) or a fracture of the medial wall and a minor associated fracture of the floor of the orbit: this was defined as a $<1 \mathrm{~cm}^{2}$ fracture located medially to the infraorbital canal ( 3 more patients).

2. An actual or predictable clinical consequence of the fracture as defined by:

a) the presence of diplopia and/or enophthalmos equal to or greater than $2 \mathrm{~mm}$;

b) a fracture involving more than $1 \mathrm{~cm}^{2}$ or $50 \%$ of the medial wall with or without an associated minor fracture of the orbital floor.

\section{Exclusion criteria were:}

1. General contraindications to surgery (Age $>75$ years, high or very high anaesthesiologic risk)

2. An isolated fracture of the medial wall smaller than $1 \mathrm{~cm}^{2}$ or $50 \%$ of the wall itself with absence of diplopia and/or enophthalmos equal to or greater than $2 \mathrm{~mm}$.

3. A complex combined fracture of the medial wall and the orbital floor.

Every patient underwent physical exam, an ophthalmologic evaluation and a CT scan of the head before the operation.

Of the 25 patients, 21 had enophthalmos, and 7 had diplopia. Three patients presented with severe limitations in eye abduction.

\subsection{Surgical technique}

Surgery is started by placing the patient in the standard position for endoscopic sinus surgery (ESS); both eyes must be visible in the operating field. They are kept closed with one steristrip for each eye.

The procedure is initiated with a $0^{\circ}$ endoscope, and a radical anteroposterior ethmoidectomy at the involved side is carried out. Extreme care is taken while approaching the fractured lamina papyracea (Fig. 1). The swollen mucosa must be distinguished from the herniated orbital content. All fractured fragments must be removed in order to avoid pushing them back in the orbit at the time of positioning the reconstructive sheet. At the same time it is wise not to leave any residual mucosal fragments to prevent infection or mucosal retention cysts.

When all the fractured bone fragments and mucosal residuals have been removed, the following constant landmarks are identified: the superior and the inferior healthy margins of the medial wall are easily checked. We have found that the posterior apex of the fracture (theoretically the third margin) was invariably an acute angle resulting from the union of the upper and lower margins and not a distinct linear margin.

The anterior margin of the fracture is on the other side, more difficult to visualize. If there is any doubt that the posterior lacrimal crest is being reached, then this is not further skeletonized and the anterior margin is only palpated.

A flexible ruler (cut to the purpose from a sterile skin marker ruler) is then introduced in the meatus and is used to measure the anterior-posterior size of the defect (see Fig. 2).

At this point a $0.8 \mathrm{~mm}$ thick Porous high density polyethylene (Medpor) sheet is shaped exceeding the measured defect by few millimetres both in the sagittal as well as in the vertical dimensions. The shape of the prosthesis will closely resemble that of a guitar pick. Finally it is given a slight medial convexity to mimic the usual shape of the medial wall.

The sheet is introduced in the nose over the herniated content and gently pushed laterally until the fracture margins are met. Then the upper part of the sheet is pushed beyond the superior margin of the fracture with the tip of a curved sinus aspirator. The same is repeated for the lower border. The sheet becomes then self-

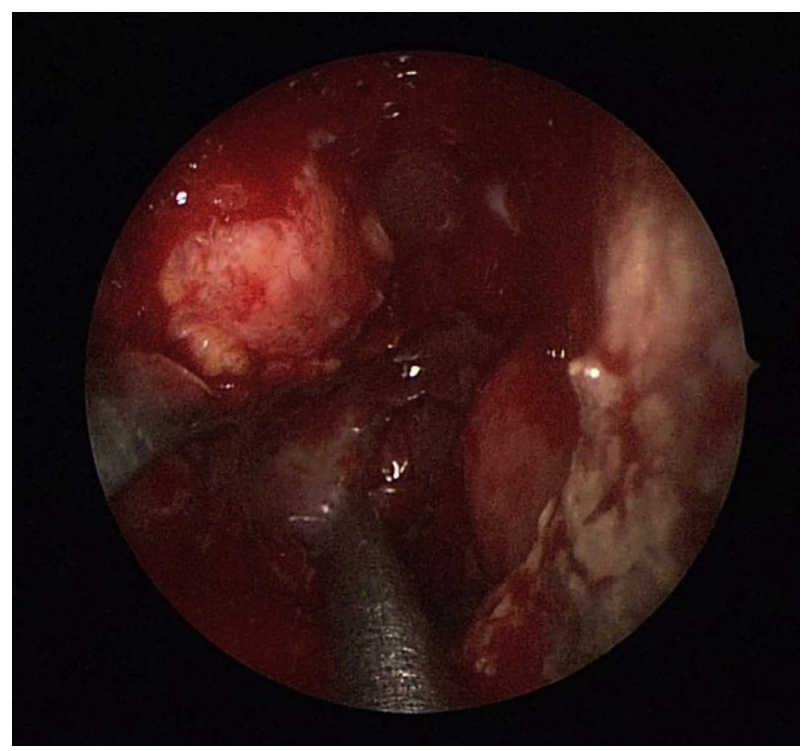

Fig. 1. Intraoperative endoscopic still showing the orbital content protruding from the fracture margin after a complete right ethmoidectomy and the removal of all bony fragments. 


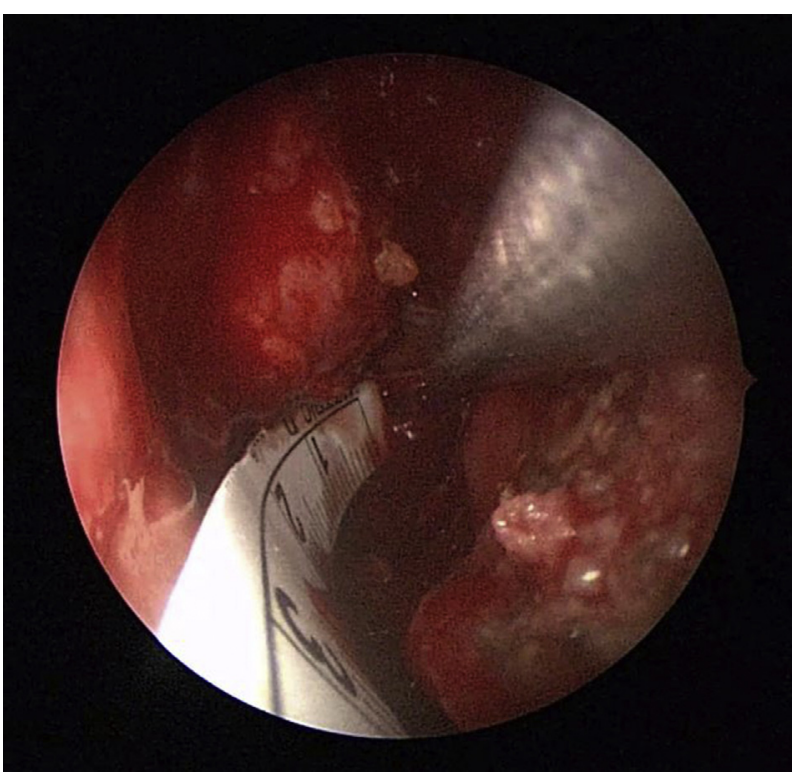

Fig. 2. Intraoperative endoscopic still showing the ruler used to measure the anterior-posterior size of the defect.

containing owing to the planned exceeding borders that will impinge on the fracture margins (see Fig. 3). Gentle pressure on the eyeball will confirm the steadiness of the reconstruction.

Frequently, during this procedure, a $45^{\circ}$ endoscope allows a better view of the field and is then preferred over the $0^{\circ}$. In the first 3 patients the middle meatus was packed with absorbable porcine gelatin sheets (Spongostan special, Ethicon, Johnson \& Johnson, Edinburgh, UK), and a single nasal pack (Merocel, Medtronic, Minneapolis, MI, USA). However, starting from patient 4 we did not put anything in the middle meatus because this allows for much clearer postoperative CT images and does not detract anything in terms of risk of haemorrhage.

The patient is administered $1 \mathrm{~g}$ i.v. cefazolin intraoperatively and t.i.d. $1 \mathrm{~g}$ oral amoxicillin clavulanate for 5 days postoperatively. On POD 2 a plain CT scan is done to confirm a correct reconstruction and to rule out any potential local complications. The same day, after a standard ophthalmological evaluation, the patient is discharged.

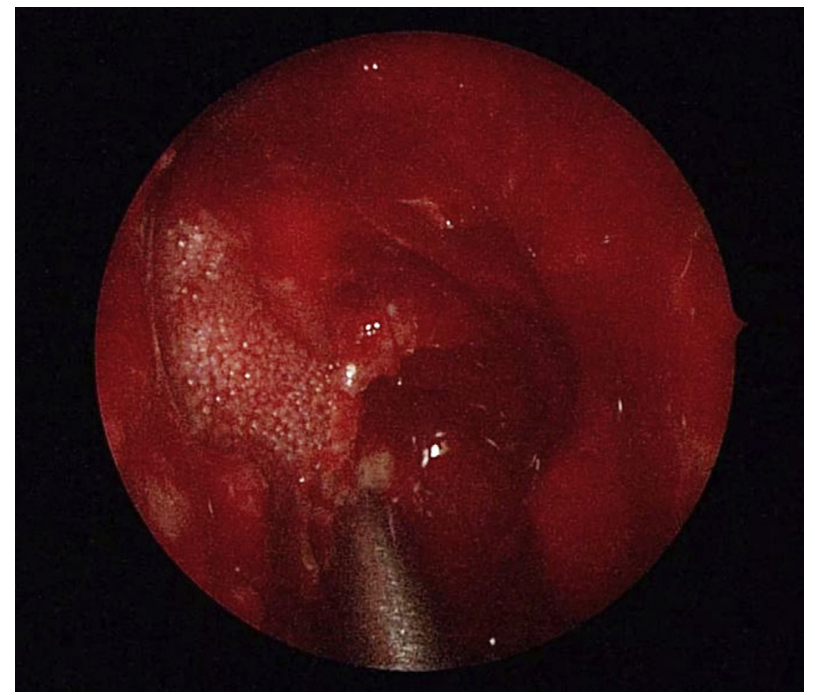

Fig. 3. Intraoperative endoscopic still showing the Medpor sheet encased into the fracture margins, completely correcting the orbital content herniation.

\subsection{Follow up}

All patients were seen again on POD 7, 30 and 180. A simple fiberoptic inspection was carried out, and the projection of the eyeball and the binocular vision were checked (see Fig. 4).

\section{Results}

The average time for each surgical procedure was $40 \mathrm{~min}$, ranging from 30 to $60 \mathrm{~min}$.

No perioperative complications were registered. After observing the first patients, we noticed a very high degree of subjective comfort. This was confirmed verbally by all patients. However, we did not keep written track of these results and we can thus not quantify this aspect. The immediate morphological and functional results were good: in all patients, a complete resolution of the enophthalmos was achieved. Primary gauze showed absence of residual diplopia in all patients. In 9 patients, in the immediate postoperative period, there was a residual diplopia in the extreme lateral gaze. At the mid and long term follow up, no residual diplopia in any position was noted (see Figs. 5-24). Endoscopic examinations showed partial reepithelization of the meshes at POD 30. At POD 180 all the meshes were covered by mucosa. No infections or extrusions were noted.

\section{Discussion}

Fractures of the medial wall of the orbit are, according to some recent papers, at least as frequent as fractures of the orbital floor (Choi et al., 2015). However, there has been considerably more literature on the treatment of the latter. It may hold true that fractures of the floor have (usually) a greater impact on form and function of the orbit as a whole (Higashino et al., 2011). However, fractures of the medial wall that cause diplopia or enophthalmos or fractures greater than $1 \mathrm{~cm}^{2}$ or than $50 \%$ of the wall carry a similar burden.

Fractures of the medial wall are more difficult to treat than fractures of the floor, however. There are more delicate anatomical structures that have to be identified and respected, such as the lacrimal apparatus, the ethmoidal arteries, the medial rectus and

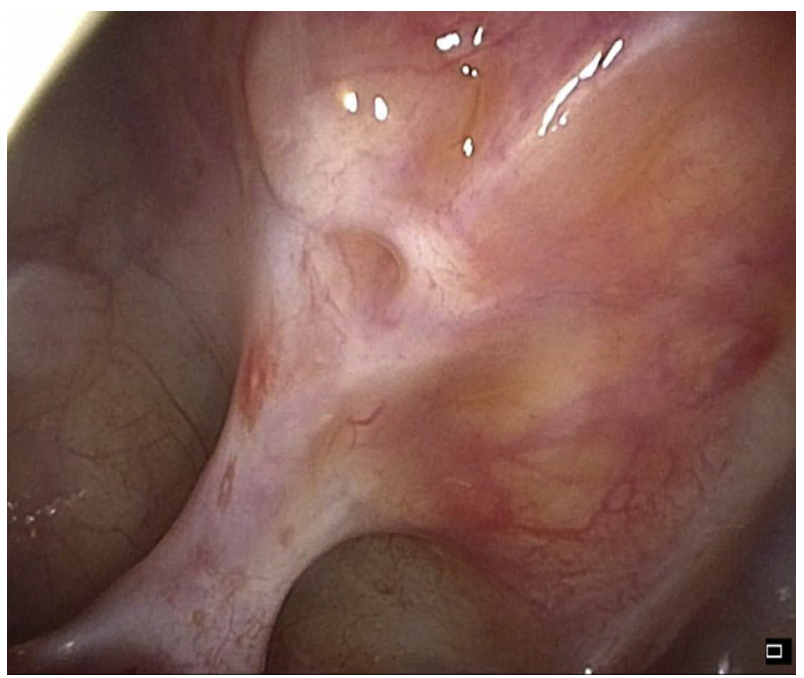

Fig. 4. Postoperative endoscopic still showing the complete reepithelization of the Medpor sheet. No signs on infection or extrusion can be seen and the mucosa lining the sheet appears as completely indistinguishable from the surrounding healthy ethmoid mucosa. 


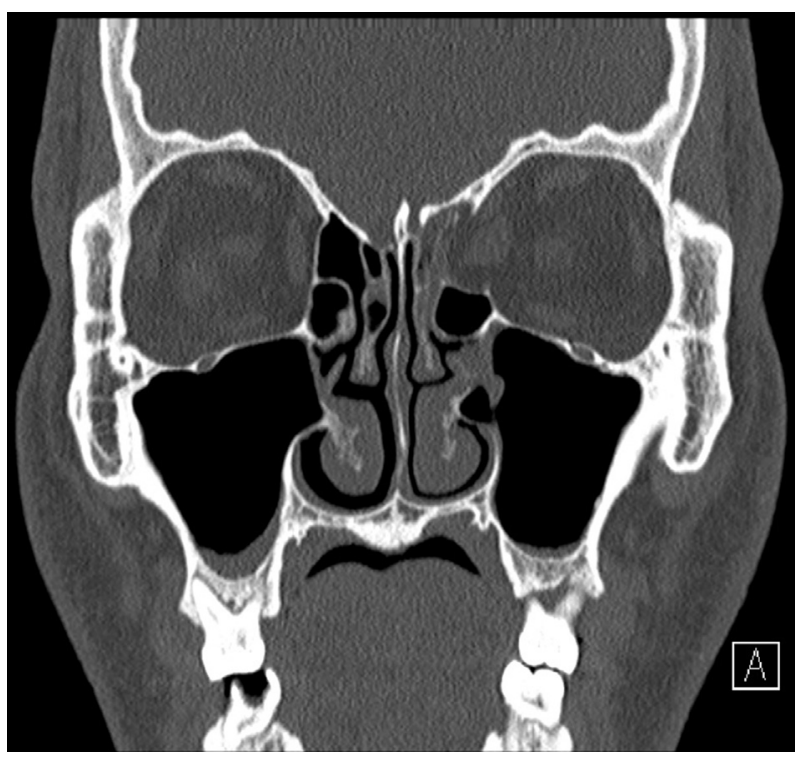

Fig. 5. Coronal plain head CT image (Patient A) showing left medial orbital wall fracture. The orbital soft tissues are herniating toward the ethmoid together with the left medial rectus muscle.

the greater oblique muscle. Even more so, the anterior skull base is separated from the orbit by thin bony sheaths only. Thus improper approaches to fractures of the medial wall are far more hazardous and may potentially lead to severe or disastrous complications (Colletti et al., 2012, 2015). This may be the real reason why treatment of fractures of the medial wall of the orbit have long been left untreated, with obvious consequences.

Traditionally, when these were treated, a Lynch skin incision was adopted. This skin incision, if used to access the medial wall of

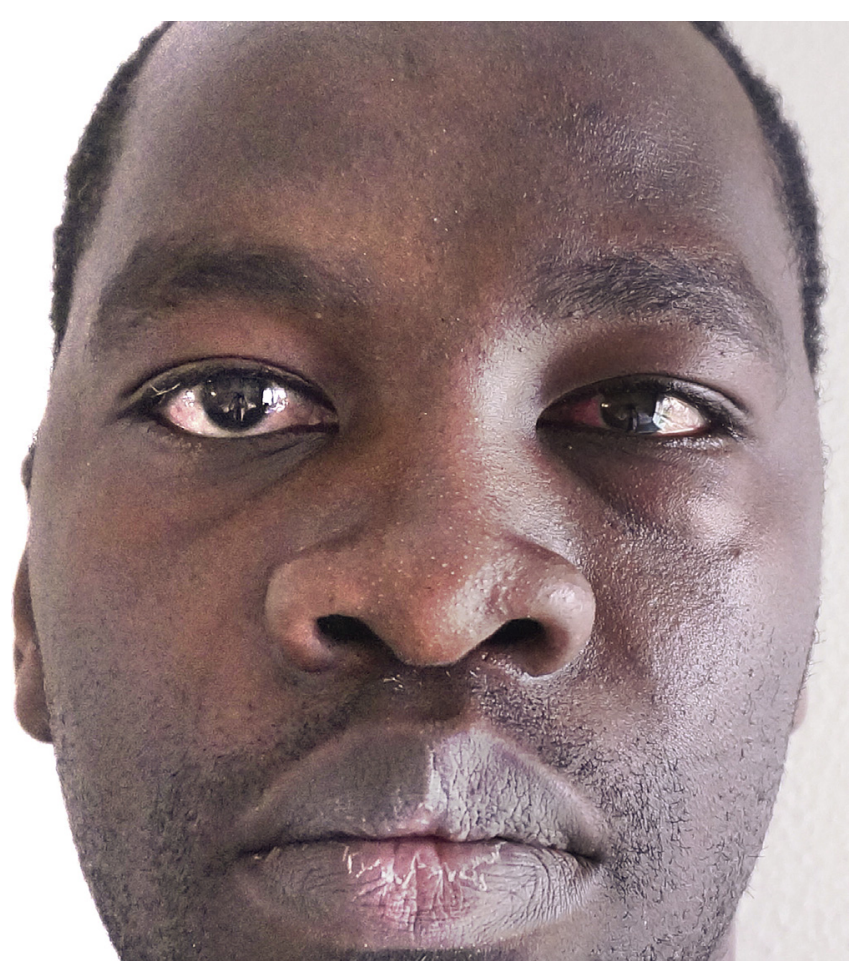

Fig. 6. Preoperative photograph of patient A. Left enophthalmos and left eye chemosis are clearly visible.

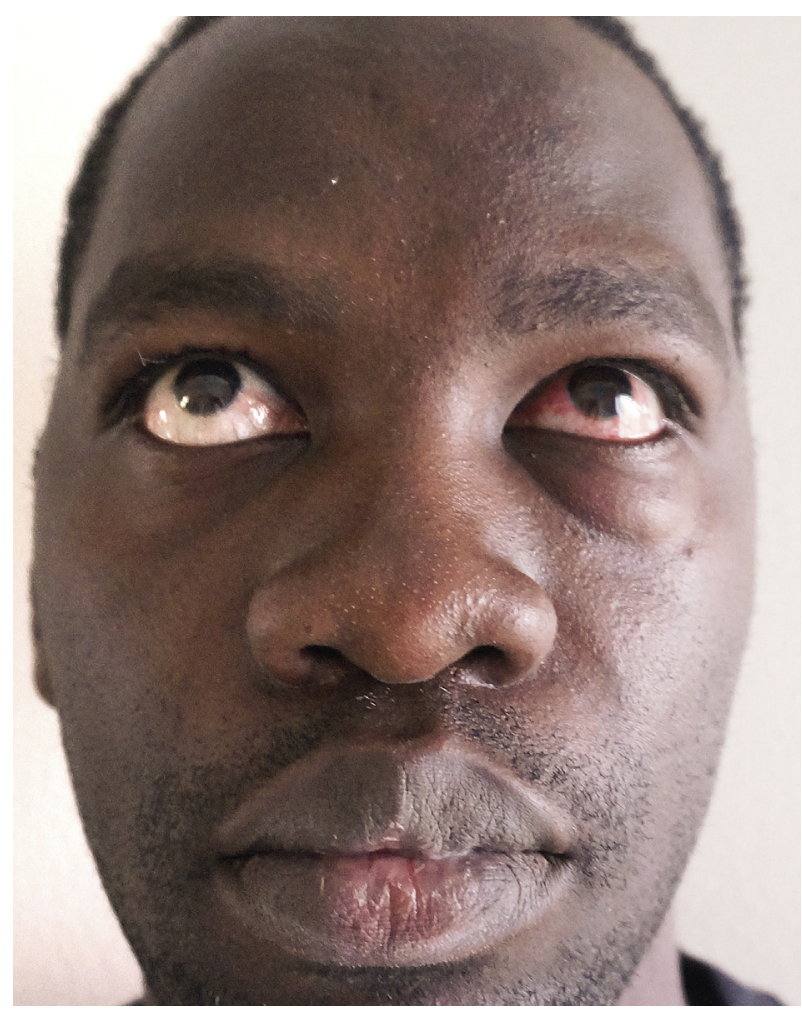

Fig. 7. Preoperative photograph of patient A. Left enophthalmos, slight left ophthalmoplegia and left eye chemosis are clearly visible.

the orbit, contemplates the detachment of the medial canthal ligament (Boyette et al., 2015). Postoperative malpositions or unsightly scars, unsurprisingly, have been not infrequent.

Other transpalpebral accesses have been developed such as the subciliary and the subtarsal approach. These guarantee better results in terms of skin scar. Still they allow for just a limited view of the operative field (especially the superior and the posterior part of the medial wall) and leave the mark of a scar.

Thereafter, in the pursuit of a more respectful approach, trasnconjunctival accesses have been described (vide supra).

Still, all external approaches require a variable degree of manipulation of the conjunctiva, the cornea, the lacrimal apparatus

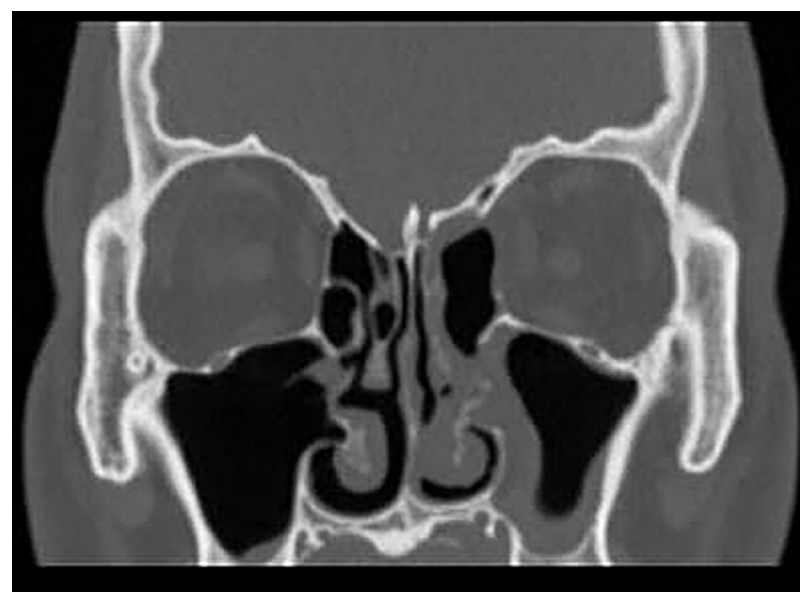

Fig. 8. Postoperative coronal plain head CT image (patient A). The left orbital content herniation is completely corrected by the Medpor sheet and the orbits are symmetric. Standard left radical ethmoidectomy results can be also seen as per surgical approach. 


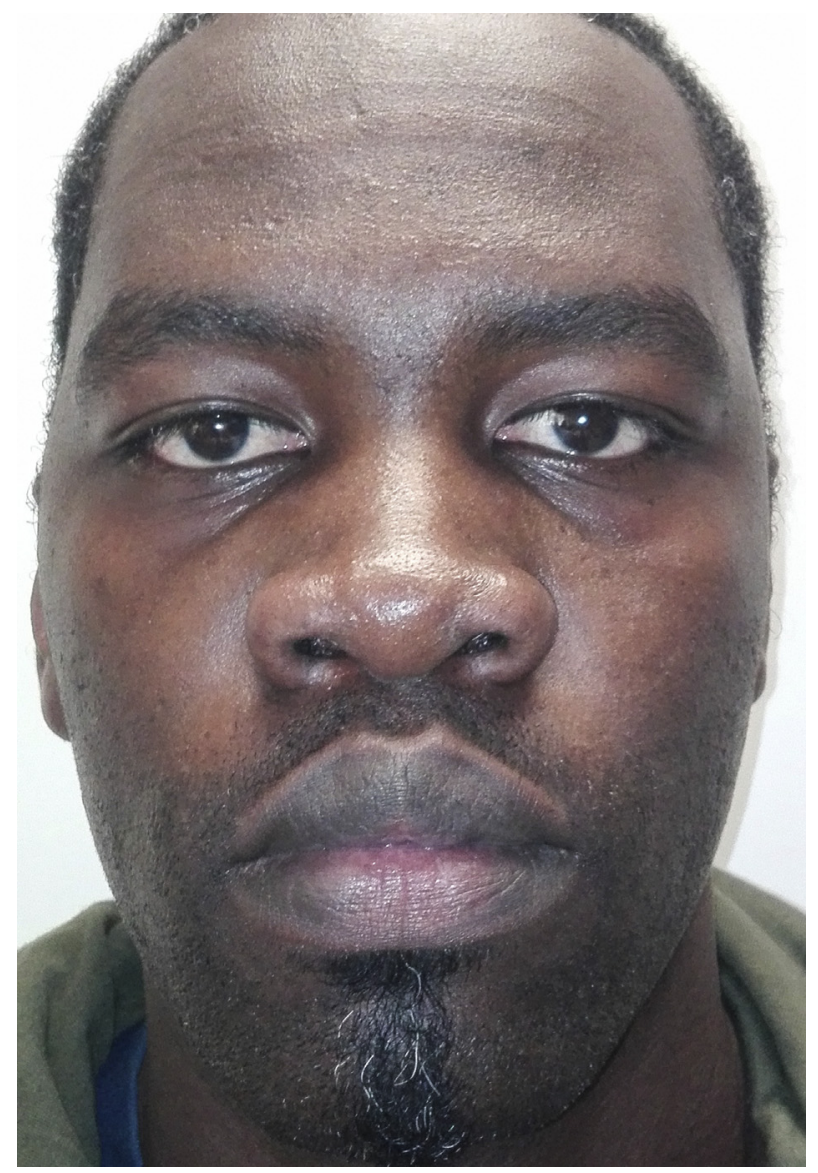

Fig. 9. Postoperative photograph of patient A. No residual enophthalmos or ophthalmoplegia can be seen. Eye symmetry is restored.

and allow for a less than desirable exposure of the surgical field (Boyette et al., 2015).

The early postoperative period after such accesses is far from symptom free. Significant chemosis, epiphora, blurred vision, minor corneal injuries and diplopia can be observed (Boyette et al., 2015).

Yamaguchi first reported an endoscopic endonasal repair of blowout fractures of the medial wall of the orbit (Nobumasa et al., 1991).

An endoscopic approach to medial wall fractures presents obvious advantages. One might argue that the first advantage is to spare the patient a scar. Although this is true, we believe that the main advantage of the endoscopic approach consists in avoiding contact with the orbital content and the lids. Thus, no epiphora and a much lower degree of early postoperative diplopia, blurred vision, and pain are expected (and, in our experience, observed).

Still, conventional endoscopic techniques traded these advantages with the discomfort (and potential complications) associated with nasal packing (Copelli et al., 2015). In most reports, 15-30 days of packing was required to hold in place a silicone sheet (or another material) that was used to reduce and keep in place the herniated orbital content (Copelli et al., 2015).

Other than these drawbacks, indirect control of the fracture such as that allowed by these endoscopic techniques may lead to imperfect results, since the soft tissues are not visually controlled, not even with the aid of navigation, which has been invoked by some authors (Copelli et al., 2015). More, the soft tissues may still re-herniate at the time of the removal of the tampons: some

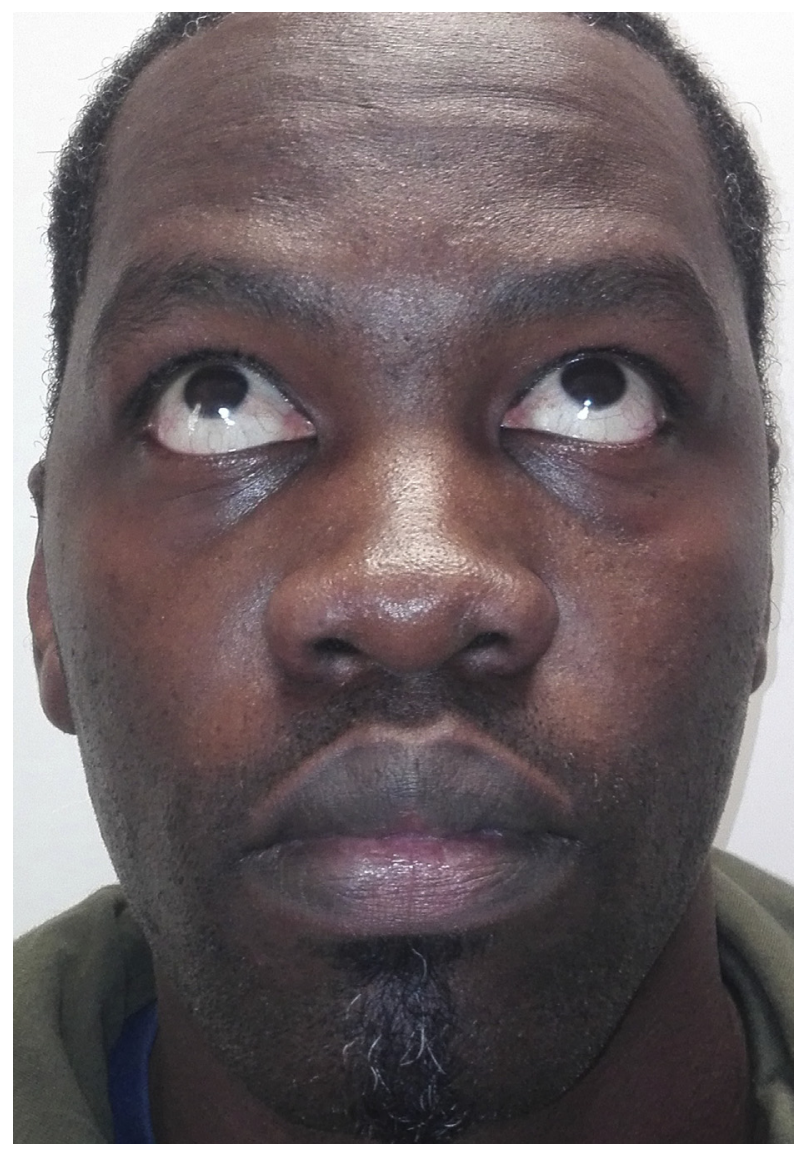

Fig. 10. Postoperative photograph of patient A. No residual enophthalmos or ophthalmoplegia can be seen. Eye symmetry is restored.

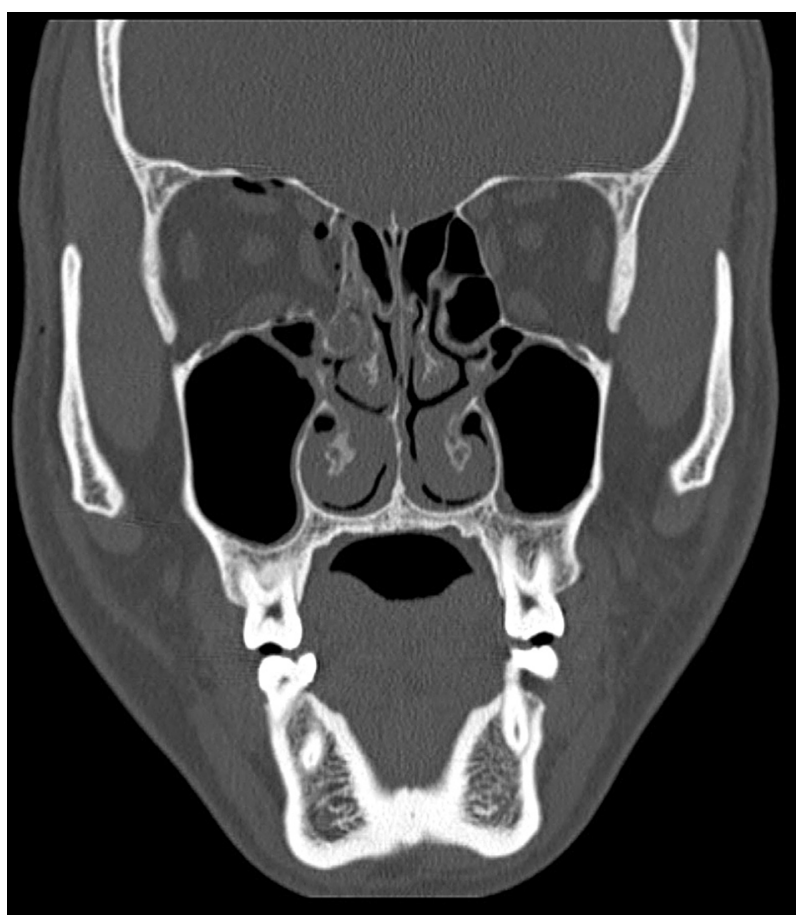

Fig. 11. Coronal plain head CT image (Patient B) showing right medial orbital wall fracture. The orbital soft tissues are herniating toward the ethmoid together with the inferior portion of the right medial rectus muscle. 


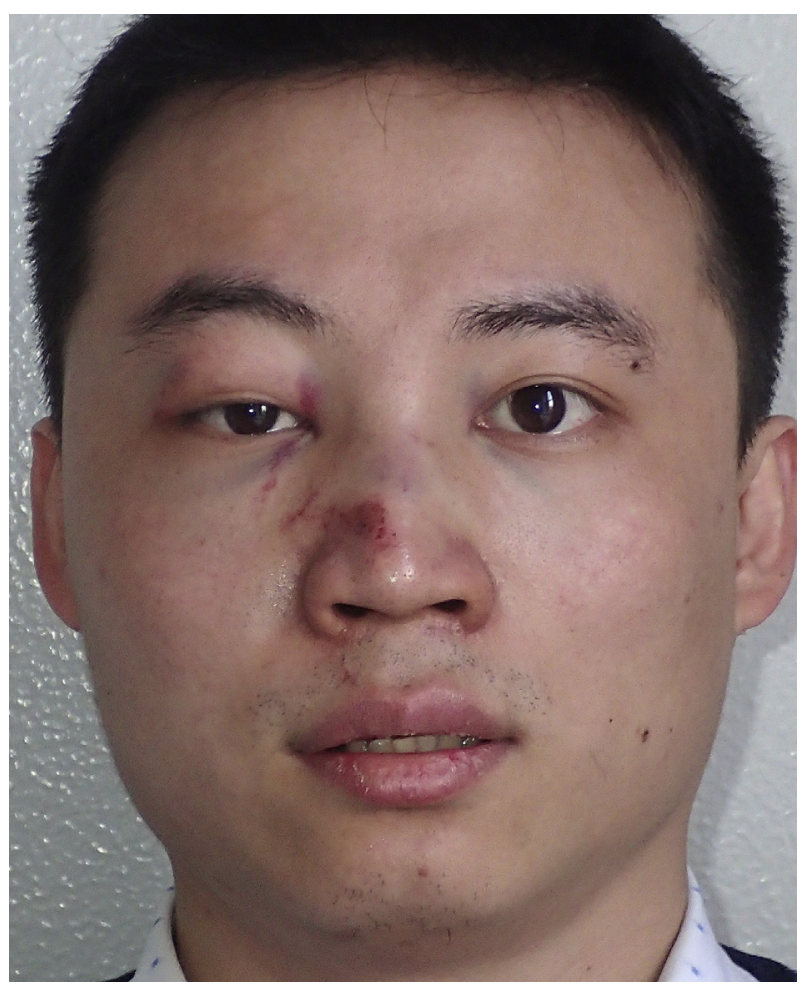

Fig. 12. Preoperative photograph of patient B. Right enophthalmos can be seen.

authors stated that for optimal results these should be maintained for 2 months and that 1 month would not be enough (Sanno et al., 2003).

It seemed logical for us to conjoin the advantages of both worlds: avoid manipulating the orbital content (typical to the endoscopic approach) and reconstruct the fractured wall (usually obtained with an external approach).

Although there have been episodic reports in the literature of authors trying to do this with various methods and materials

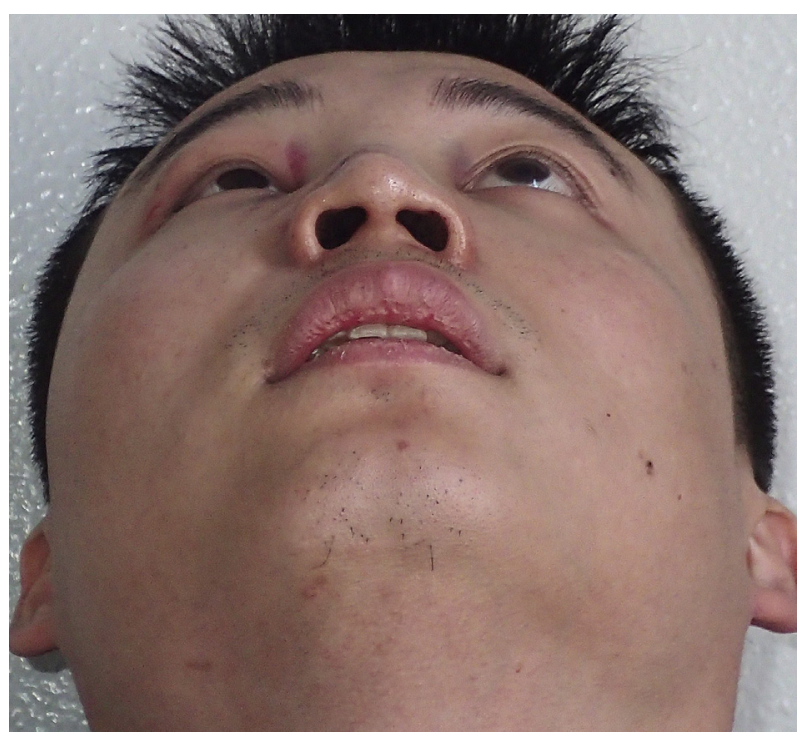

Fig. 13. Preoperative photograph of patient B. Tight enophthalmos and slight right ophthalmoplegia can be seen.

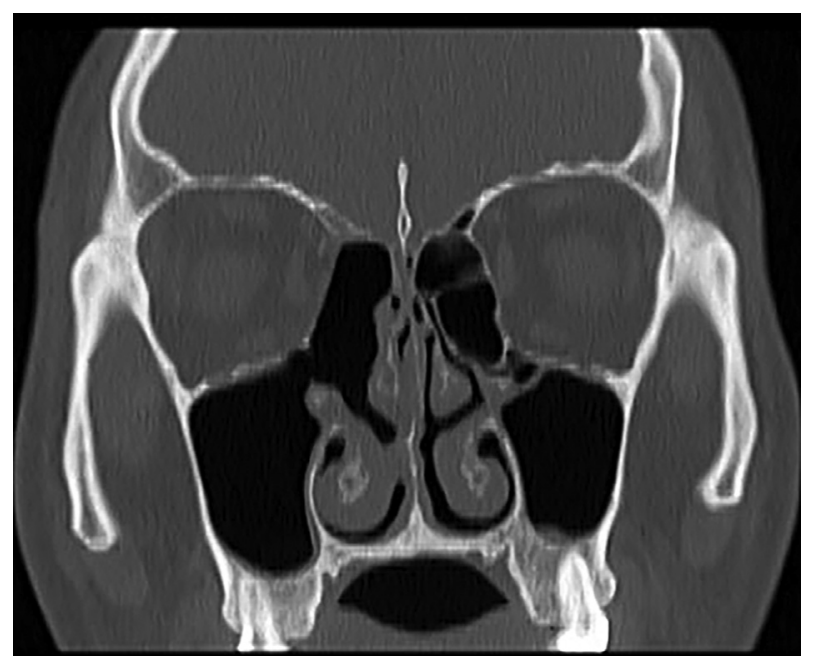

Fig. 14. Postoperative coronal plain head CT image (patient B). The right orbital content herniation is completely corrected by the Medpor sheet and the orbits are symmetric. No residual muscle herniation can be seen.

(the nasal septum, mainly), the results have been not fully convincing and the techniques have not been widely adopted ( $\mathrm{Li}$ et al., 2009).

One advantage of the technique presented here leans on the use of a polyethylene sheet. This has been widely used to repair the floor of the orbit and has proven reliable (Ram and Shadab, 2010; Kang et al., 2015). This material is strong enough while highly moldable (Lee et al., 2005). The different grade of porosity allows the ingrowth of fibrovascular cells into the implant, preventing the

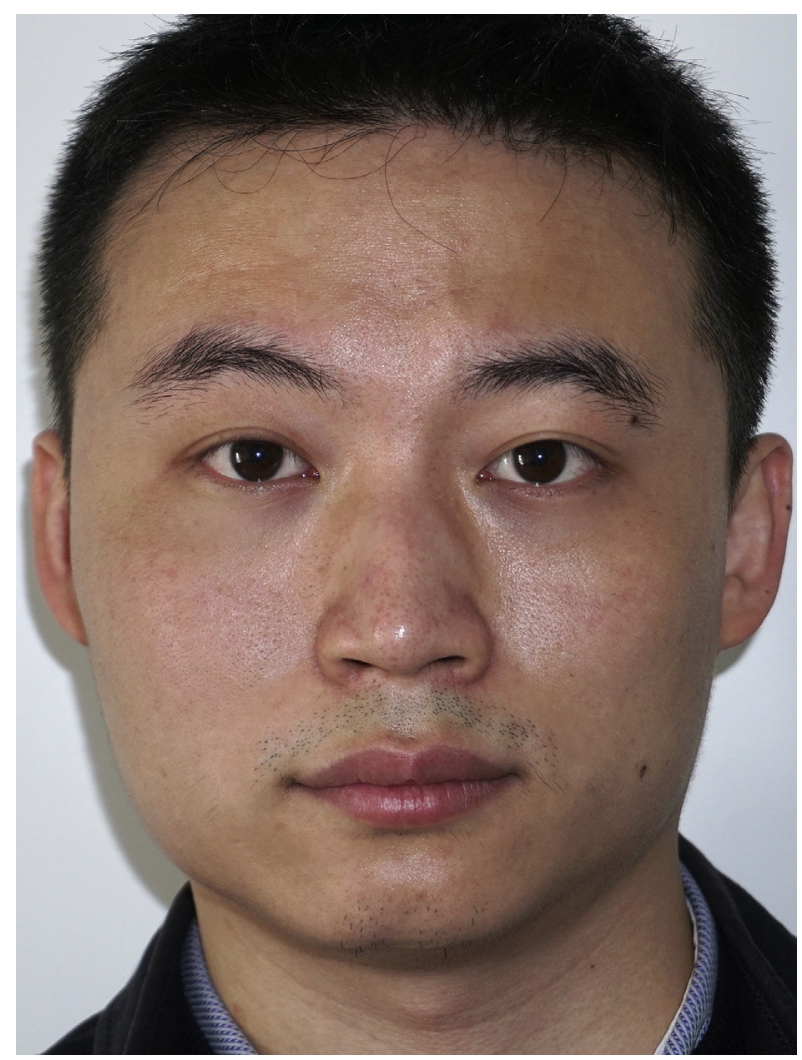

Fig. 15. Postoperative photograph of patient B. No residual enophthalmos or ophthalmoplegia can be seen. Eye symmetry is restored. 


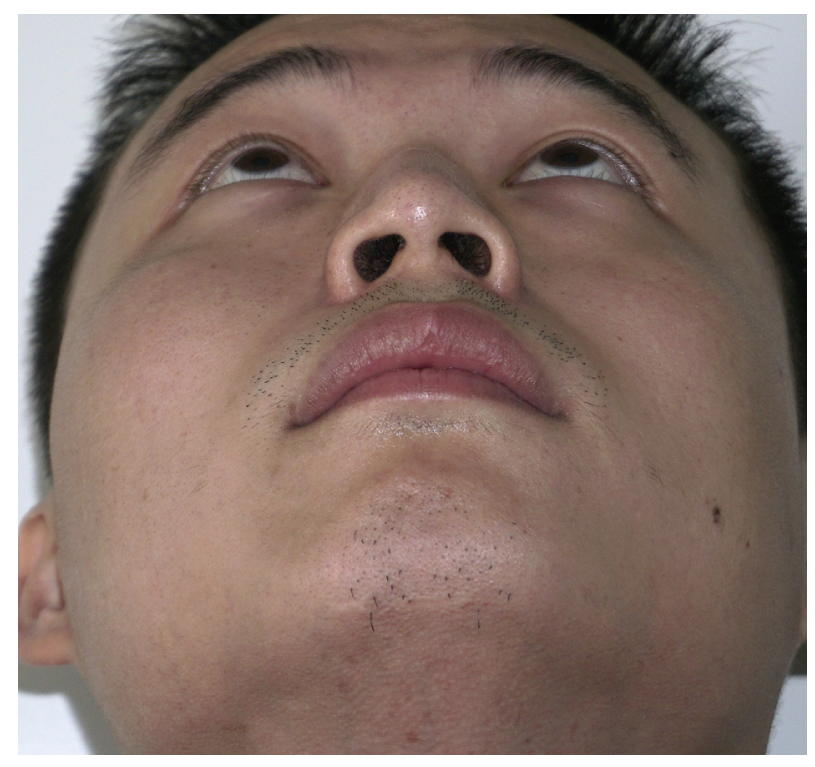

Fig. 16. Postoperative photograph of patient B. No residual enophthalmos or ophthalmoplegia can be seen. Eye symmetry is restored.

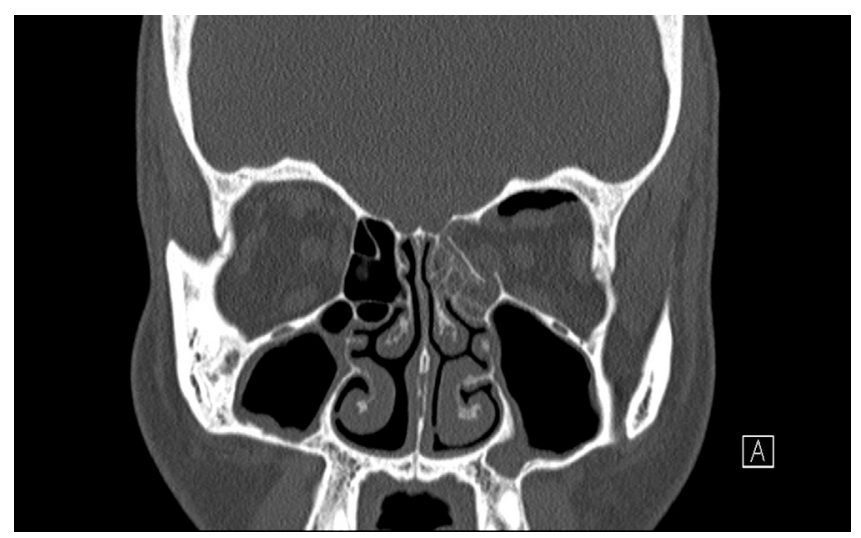

Fig. 17. Coronal plain head $C T$ image (Patient $C$ ) showing left medial orbital wall fracture. The orbital soft tissues are herniating toward the ethmoid together with the inferior portion of the left medial rectus muscle. Air can be seen in the cranial portion of the orbit.

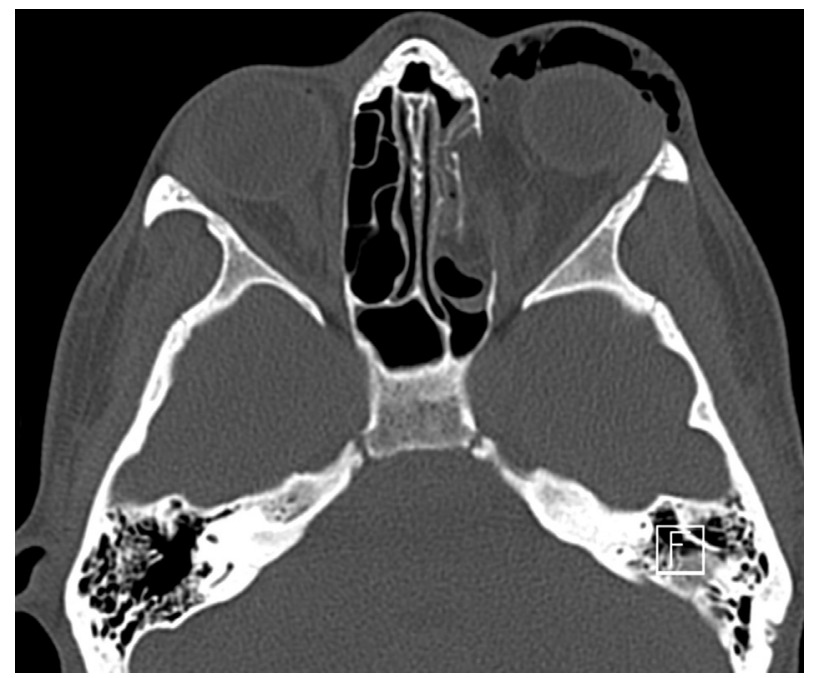

Fig. 18. Axial plain head $\mathrm{CT}$ image (Patient $\mathrm{C}$ ) showing the whole length of the left medial orbital wall fracture.

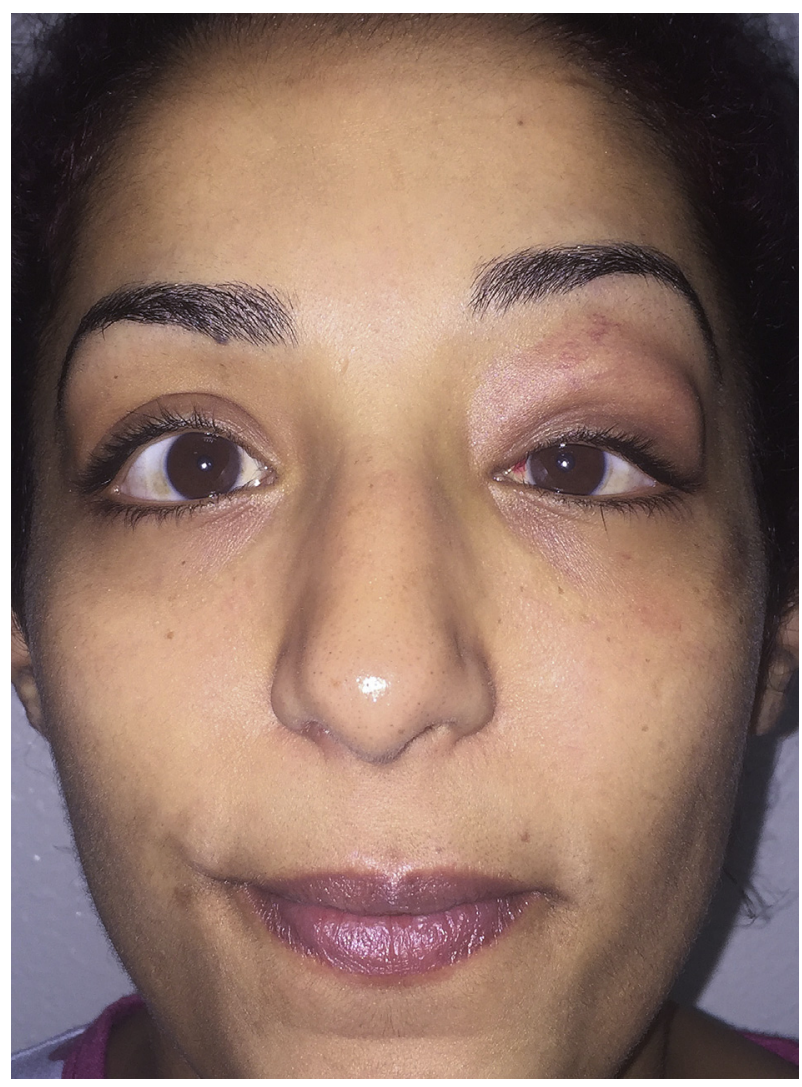

Fig. 19. Preoperative photograph of patient $C$. The image shows left ophthalmoplegia and left enophthalmos.

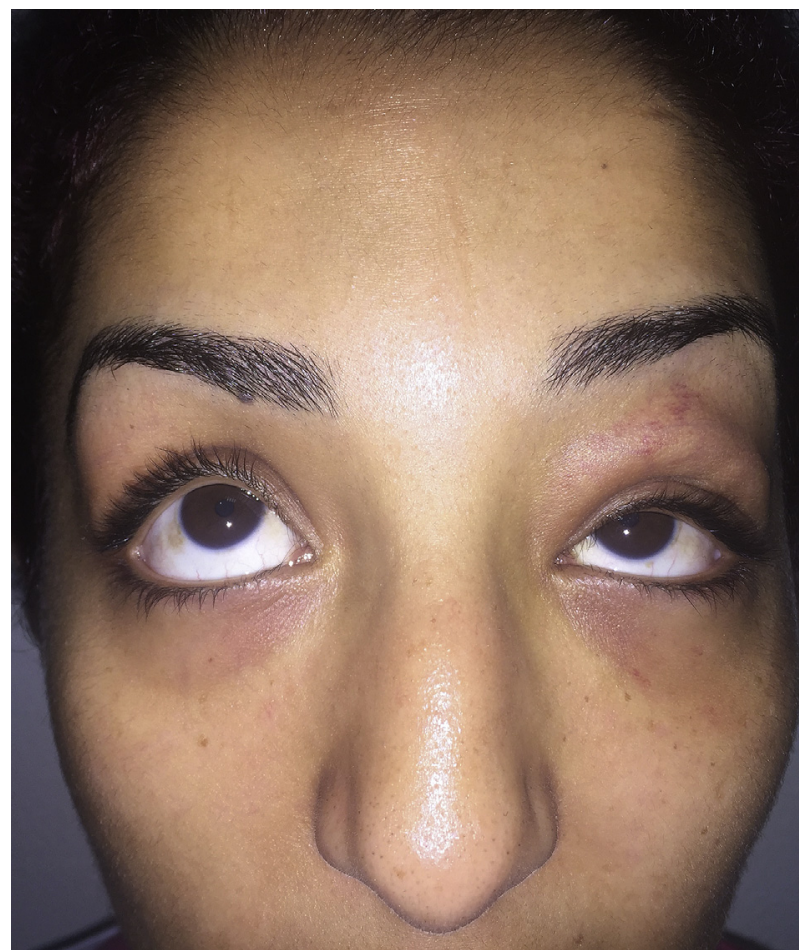

Fig. 20. Preoperative photograph of patient $C$. The image shows left ophthalmoplegia and left enophthalmos. 


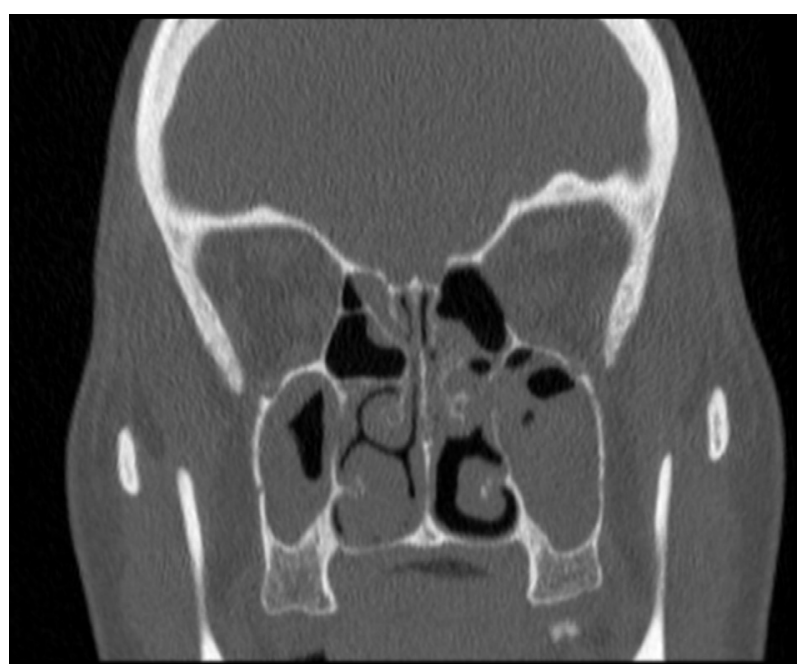

Fig. 21. Postoperative coronal plain head $C T$ image (patient $C$ ). The left orbital content herniation is completely corrected by the Medpor sheet and the orbits are symmetric. Standard left radical ethmoidectomy results can be also seen as per surgical approach.

formation of capsules and enhancing its long term stability (Lee et al., 2005). Moreover, due to a high rate of periimplant vascularisation, Medpor is more resistant to infection than other porous implants, such as expanded polytetrafluoroethylene.

Accordingly, in our case series the Medpor sheets have proven effective in obtaining excellent morphological and functional results and contemporarily have not been prone to any complication. No infections have been registered and all the sheets were covered with mucosa after 6 months.

Further improvements in our technique could stem from employing special adsorbable polydioxanon (PDS) or polylactide (PLA) foils, which could provide middle-term support to the fracture, while correctly guiding the fibrosis process. Another feasible option would be simply reducing the mesh thickness, given the

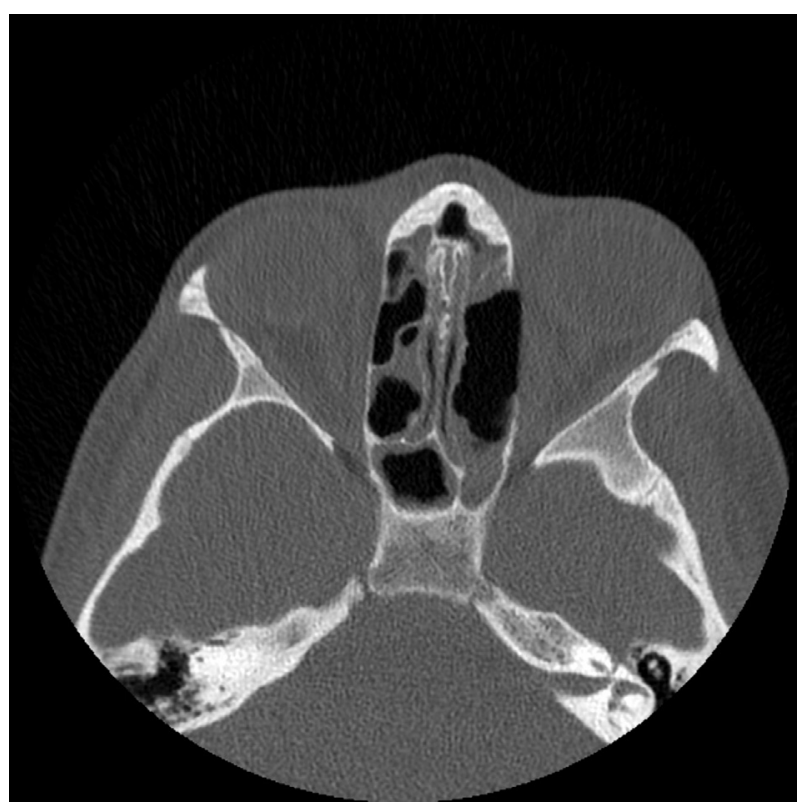

Fig. 22. Postoperative axial plain head $C T$ image (patient $C$ ). The left orbital content herniation is completely corrected by the Medpor sheet along the whole anterior-posterior length of the fracture.

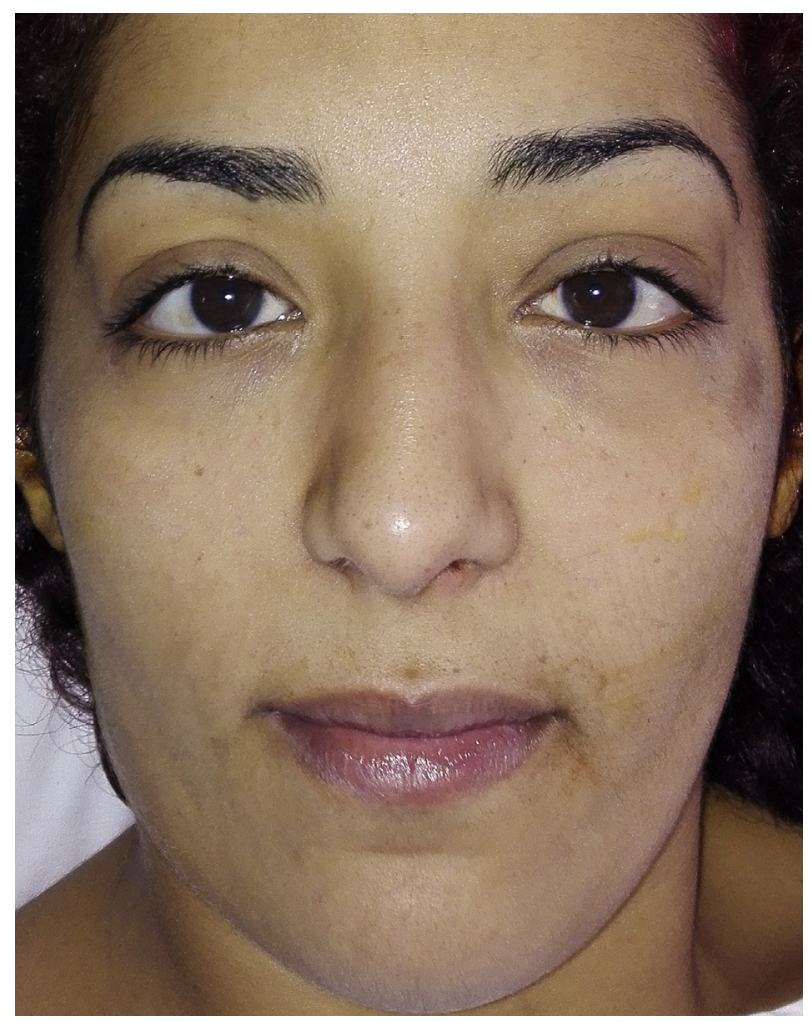

Fig. 23. Postoperative photograph of patient C. No residual enophthalmos or ophthalmoplegia can be seen. Eye symmetry is restored.

minimal mechanical load of the fracture, thus reducing the sheer volume of foreign material inserted in the orbit.

It must be noted that, after having published the preliminary report in 2016, our technique has received further validation, both in

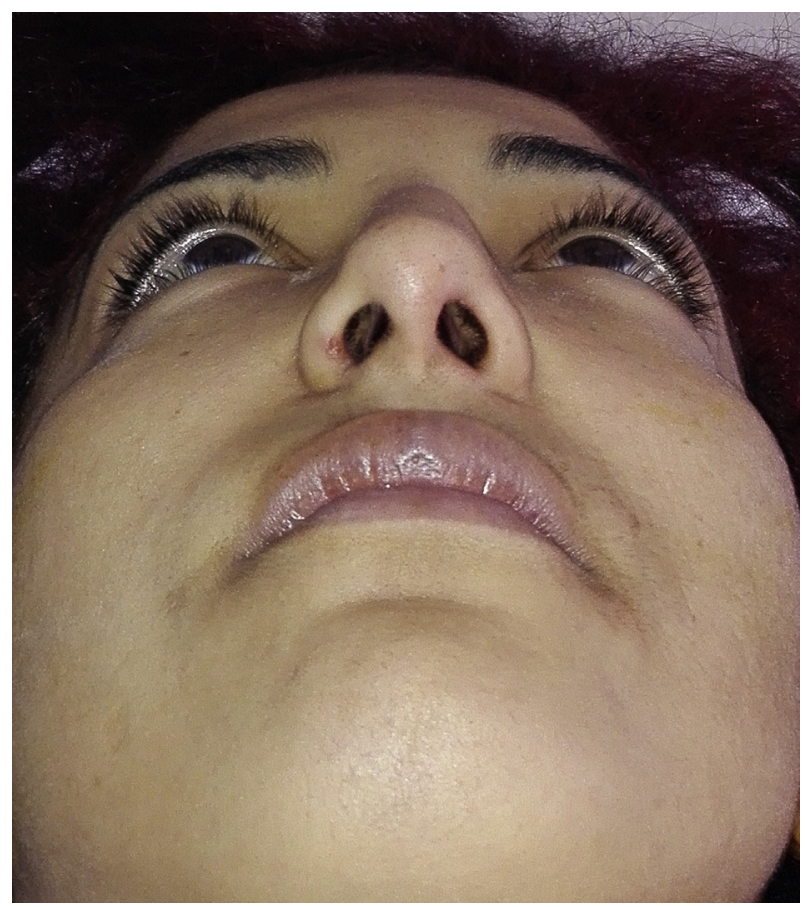

Fig. 24. Postoperative photograph of patient C. No residual enophthalmos or ophthalmoplegia can be seen. Eye symmetry is restored. 
our group (where it is being used for complex reconstructions after removal of orbital masses), and by other authors, giving solid results with only minor modifications by other specialist groups, thus reinforcing our observations (Colletti et al., 2017; Procacci et al., 2017).

\section{Conclusion}

Our experience with 25 patients allows us to surmise that:

a) Treating medial wall fractures by means of an exclusive transnasal approach while still reconstructing the fractured wall using a Medpor sheet is feasible

b) The quality of the anatomical restoration is similar or superior to that achievable with traditional approaches

c) The tolerability of the procedure may be far superior as compared to external accesses

d) The results are predictable and stable and the complication rate is exceedingly low

For these reasons, in our department, endoscopically treating fractures of the medial wall has become the standard.

It is, however, true that this is a delicate surgery and that the operator must be very confident with endoscopic surgery of the nose, with the anatomy of a fractured orbit, and with the modelling of the implant in order to maximize results and keep complication rates low.

\section{References}

Bartoli D, Fadda MT, Battisti A, Cassoni A, Pagnoni M, Riccardi E, et al: Retrospective analysis of 301 patients with orbital floor fracture. J Craniomaxillofac Surg 43: 244-247, 2015

Boyette JR, Pemberton JD, Bonilla-Velez J: Management of orbital fractures: challenges and solutions. Clin Ophthalmol 9: 2127-2137, 2015

Bullock JD, Warwar RE, Ballal DR, Ballal RD: Mechanisms of orbital floor fracture: a clinical, experimental, and theoretical study. Trans Am Ophthalmol Soc 97: $87-113,1999$

Choi KE, Lee J, Lee H, Chang M, Park M, Baek S: The paradoxical predominance of medial wall injuries in blowout fracture. J Craniofac Surg 26: e752-e755, 2015

Colletti G, Valassina D, Rabbiosi D, Pedrazzoli M, Felisati G, Rossetti L, et al: Traumatic and iatrogenic retrobulbar hemorrhage: an 8-patient series. J Oral Maxillofac Surg 70: e464-e468, 2012
Colletti G, Fogagnolo P, Allevi F, Rabbiosi D, Bebi V, Rossetti L, et al: Retrobulbar hemorrhage during or after endonasal or periorbital surgery. J Craniofac Surg 26: 897-901, 2015

Colletti G, Pipolo C, Lozza P, Felisati G, Allevi F, Biglioli F, et al: Orbital medial wall fractures: purely endoscopic endonasal repair with polyethylene implants. Clin Otolaryngol. https://doi.org/10.1111/coa.12675, 2016 [Epub ahead of print]

Colletti G, Saibene AM, Pessina F, Duvina M, Allevi F, Felisati G, et al: A shift in the orbit: immediate endoscopic reconstruction after transnasal orbital tumors resection. J Craniofac Surg. https://doi.org/10.1097/SCS.0000000000003879, 2017 [Epub ahead of print]

Copelli C, Manfuso A, d'Ecclesia A, Catanzaro S, Cassano L, Pederneschi N, et al: Endoscopic transnasal approach and intraoperative navigation for the treatment of isolated blowout fractures of the medial orbital wall. J Craniomaxillofac Surg 43: 1974-1978, 2015

Damgaard OE, Larsen CG, Felding UA, Toft PB, von Buchwald C: Surgical timing of the orbital 'blowout' fracture: a systematic review and meta-analysis. Otolaryngol Head Neck Surg 155: 387-390, 2016

Duane TD, Schatz NJ, Caputo AR: Pseudo-Duane's retraction syndrome. Trans Am Ophthalmol Soc 74: 122-132, 1976

Higashino T, Hirabayashi S, Eguchi T, Kato Y: Straightforward factors for predicting the prognosis of blow-out fractures. J Craniofac Surg 22: 1210-1214, 2011

Jin HR, Yeon JY, Shin SO, Choi YS, Lee DW: Endoscopic versus external repair of orbital blowout fractures. Otolaryngol Head Neck Surg 136: 38-44, 2007

Joseph JM, Glavas IP: Orbital fractures: a review. Clin Ophthalmol 5: 95-100, 2011

Kahn AM, Varvares MA: Traditional approaches to the orbit. Otolaryngol Clin North Am 39: 895-909, 2006

Kang N, Song SH, Kyung H, Oh SH: Medpor implant fixation using fibrin glue in the treatment of medial orbital wall fracture. J Craniofac Surg 26: 1361-1364, 2015

Kempton SJ, Cho DC, Thimmappa B, Martin MC: Benefits of the retrocaruncular approach to the medial orbit: a clinical and anatomic study. Ann Plast Surg 76: 295-300, 2016

Kim YH, Park Y, Chung KJ: Considerations for the management of medial orbital wall blowout fracture. Arch Plas Surg 43: 229-236, 2016

Lee S, Maronian N, Most SP, Whipple ME, McCulloch TM, Stanley RB, et al: Porous high-density polyethylene for orbital reconstruction. Arch Otolaryngol Head Neck Surg 131: 446-450, 2005

Li H, Den W, Mo L, Yang X, Jiang D: Prosthetic treatment of blow-out fracture in medial orbital wall with nasoseptal cartilage under nasal endoscope 23: 394-396, 2009

Lynch RC: The technique of a radical frontal sinus operation which has given me the best results. Laryngoscope 31: 1-5, 1921

Nobumasa Y, Shuichi A, Hiroki M, Yutaka U: Endoscopic endonasal technique of the blowout fracture of the medial orbital wall. Contemp Sinus Surg 2: 269-274, 1991

Procacci P, Trevisiol L, Nocini PF, Favero V, D'Agostino A: Modified technique for endoscopic endonasal reduction of medial orbital wall fracture using an absorbable packing. Oral Maxillofac Surg 21: 91-98, 2017

Ram H, Shadab RKS: Efficacy of iliac crest vs. Medpor in orbital floor reconstruction. J Maxillofac Oral Surg 9: 134-141, 2010

Sanno T, Tahara S, Nomura T, Hashikawa K: Endoscopic endonasal reduction for blowout fracture of the medial orbital wall. Plast Reconstr Surg 112: 1228-1237, 2003 